\title{
Criminologie
}

\section{« 99 \% des suicides sont tragiques, nous nous battons pour le $1 \%$ qui reste »}

\author{
Des esprits sains dans des corps malades et l'activisme du droit \\ à la mort
}

\author{
"99 \% of suicides are tragic; we're fighting for the other $1 \%$ " \\ "Sound minds" and unsound bodies in right to die activism \\ "El 99 \% de los suicidios son trágicos, nosotros luchamos por el \\ $1 \%$ que queda” \\ Unas "mentes sanas" en unos cuerpos enfermos y el activismo \\ del derecho a la muerte
}

\section{Ari Gandsman}

Volume 51, numéro 2, automne 2018

Prise en charge du suicide : entre crime, troubles mentaux et droit de mourir

URI : https://id.erudit.org/iderudit/1054239ar

DOI : https://doi.org/10.7202/1054239ar

Aller au sommaire du numéro

Éditeur(s)

Les Presses de l’Université de Montréal

ISSN

0316-0041 (imprimé)

1492-1367 (numérique)

Découvrir la revue

Citer cet article

Gandsman, A. (2018). « 99 \% des suicides sont tragiques, nous nous battons pour le $1 \%$ qui reste " : des esprits sains dans des corps malades et l'activisme du droit à la mort. Criminologie, 51(2), 167-188.

https://doi.org/10.7202/1054239ar
Résumé de l'article

À partir de recherches ethnographiques menées auprès d'activistes du droit à la mort, cet article cherche à relever et à analyser les divisions de nature conceptuelle qui structurent les arguments des activistes. D’une part, des activistes soutiennent que la mort médicalement assistée doit être rigoureusement distinguée de l'acte du suicide. Pour ce faire, ils font référence à la notion « d'esprit sain » dans un contexte où le corps est malade, faisant ainsi appel au caractère fondamentalement rationnel d'un processus décisionnel fondé sur la volonté dans le choix. Ils distinguent un tel choix des suicides, non rationnels, qui sont visiblement le résultat d'un " esprit troublé ". Toutefois, en puisant dans une base de données ethnographiques considérable et dans des entrevues, on constate que les activistes reconnaissent aussi implicitement une frontière perméable entre les deux actes. Plusieurs points de tension et d'ambiguïté surviennent d'ailleurs lorsqu'il s'agit de distinguer la mort médicalement assistée et officiellement approuvée d'un acte de suicide. Dans l'analyse de la question, le présent article cherche à explorer le noyau de la tension entre les réformistes et les branches plus radicales de l'activisme du droit à la mort. Si ceux qui aspirent à modifier la constitution actuelle demandent, parmi d'autres garde-fous, des critères d'admissibilité clairement définis en fonction de maladies terminales et incurables, d'autres activistes plus radicaux s'opposent à de telles " médicalisations de la mort » et tiennent une position plus ouverte où le droit à la mort englobe aussi les « suicides rationnels ». Enfin, notre article montrera que, même après la légalisation de la mort médicalement assistée, ces noyaux de tensions subsistent. 


\title{
« $99 \%$ des suicides sont tragiques, nous nous battons pour le $1 \%$ qui reste ॥
}

\author{
Des esprits sains dans des corps malades et \\ l'activisme du droit à la mort
}

\author{
Ari Gandsman ${ }^{1}$ \\ Professeur agrégé \\ Études sociologiques et anthropologiques \\ Université d'Ottawa \\ Ari.gandsman@uottawa.ca
}

\section{Article traduit de l'anglais au français par Florence Dubois}

RÉSUMÉ - À partir de recherches ethnographiques menées auprès d'activistes du droit $\grave{a}$ la mort, cet article cherche à relever et à analyser les divisions de nature conceptuelle qui structurent les arguments des activistes. D'une part, des activistes soutiennent que la mort médicalement assistée doit être rigoureusement distinguée de l'acte du suicide. Pour ce faire, ils font référence à la notion "d'esprit sain» dans un contexte où le corps est malade, faisant ainsi appel au caractère fondamentalement rationnel d'un processus décisionnel fondé sur la volonté dans le choix. Ils distinguent un tel choix des suicides, non rationnels, qui sont visiblement le résultat d'un "esprit troublé». Toutefois, en puisant dans une base de données ethnographiques considérable et dans des entrevues, on constate que les activistes reconnaissent aussi implicitement une frontière perméable entre les deux actes. Plusieurs points de tension et d'ambiguïté surviennent d'ailleurs lorsqu'il s'agit de distinguer la mort médicalement assistée et officiellement approuvée d'un acte de suicide. Dans l'analyse de la question, le présent article cherche à explorer le noyau de la tension entre les réformistes et les branches plus radicales de l'activisme du droit à la mort. Si ceux qui aspirent à modifier la constitution actuelle demandent, parmi d'autres garde-fous, des critères d'admissibilité clairement définis en fonction de maladies terminales et incurables, d'autres activistes plus radicaux s'opposent à de telles "médicalisations de la mort» et tiennent une position plus ouverte où le droit à la mort englobe aussi les «suicides rationnels».

1. Faculté des sciences sociales, 120, Université, Pavillon des sciences sociales, local 10005, Ottawa (Ontario), Canada, K1N 6N5.

Criminologie, vol. 51, n 2 (2018) 
Enfin, notre article montrera que, même après la légalisation de la mort médicalement assistée, ces noyaux de tensions subsistent.

MOTS CLÉS • Mort, droit à la mort, mort médicalement assistée, suicide.

\section{Introduction}

Au début de notre entrevue, Jessica parle du suicide de son père. La deuxième fois où elle le mentionne, je lui demande de m'en dire plus. Elle m'explique alors comment, après une surdose de somnifères, son père s'est jeté au bas d'une falaise. Elle me dit qu'il a tout planifié calmement, méticuleusement et dans le secret le plus total, attendant même que sa mère soit en visite chez elle pour éviter qu'elle soit celle qui découvre le corps. Pour Jessica, ce suicide est le résultat d'une dépression non diagnostiquée, bien que son père n'ait jamais montré aucun symptôme et ait toujours été un «homme de carrière» et une personne «sociable». Elle suppose que le fait de risquer de perdre son emploi à 60 ans s'ajoutant à l'arrivée de certaines marques physiologiques de vieillesse ont pu être des facteurs déterminants, mais elle ne parvient pas à s'expliquer facilement cet acte.

Binna ouvre quant à elle notre entretien en me parlant du suicide de son mari, un ancien militaire qui a servi dans l'unité des forces spéciales de Rhodésie et qui a été forcé de fuir le pays après l'indépendance du Zimbabwe. Parti en Tasmanie (Australie), il a eu de la difficulté à s'adapter à son nouveau pays. Outre le fait d'avoir perdu son statut et le prestige qui l'accompagnait, il n'a jamais réussi à trouver d'emploi et souffrait toujours des effets traumatisants de la guerre. Un an après qu'ils aient refait leur vie là-bas, Binna, surprise par le bruit d'un coup de feu, allait découvrir son mari dans le salon: il s'était tiré une balle dans la tête.

Ces deux récits de suicide m'ont été racontés dans le cadre de ma recherche ethnographique sur l'activisme du droit à la mort. Binna et Jessica sont membres de deux organismes différents militant localement pour le droit à la mort. Leurs récits étaient spontanés (je ne pose jamais de questions sur le suicide), des cas loin d'être isolés dans mes recherches auprès d'activistes du droit à la mort. Certains, comme Binna, associent directement leur expérience du suicide à leur intérêt, par la suite, pour la question de la mort assistée. D'autres, comme Jessica, sont moins affirmatifs. Mais quoi qu'il en soit, toutes deux m'intriguaient étant donné que, dans les débats publics sur ce qu'on appelle communément 
l'aide médicale à mourir ou la «mort assistée», toute référence au suicide est généralement tabou.

Dans l'activisme du droit à mourir, le suicide et la «mort assistée» sont diamétralement opposés, même si les deux actes se définissent par la décision consciente de mettre fin à sa propre vie. Un suicide est plutôt considéré comme le produit irrationnel d'une maladie mentale et demeure tabou, stigmatisé et pathologisé. La mort médicalement assistée est pour sa part le produit rationnel d'une délibération raisonnée dans le contexte de la souffrance causée par une maladie terminale, intraitable ou dégénérative. Alors que les suicides requièrent des interventions visant la prévention de l'acte, la «mort assistée» mobilise l'assistance de professionnels de la santé pour provoquer la mort. Si le suicide est décriminalisé dans presque l'ensemble du monde industrialisé, l'aide médicale à mourir est toujours le sujet de débats légaux et publics, n'ayant été légalisée au Canada que récemment, en 2016, et n'étant légale ailleurs dans le monde que dans quelques États américains, aux Pays-Bas, en Belgique, au Luxembourg, en Colombie et en Suisse. Alors que le suicide est tabou et découragé, l'aide à mourir, là où elle est autorisée, est soutenue et approuvée légalement. Par ailleurs, dans certaines parties du monde où le suicide est décriminalisé, le recours à la force physique pour empêcher quelqu'un de commettre cet acte est autorisé ${ }^{2}$.

Si les débats publics entourant l'activisme du droit à la mort m'avaient préparé à cette division claire entre la mort médicalement assistée et le suicide, mes recherches ethnographiques auprès des activistes m'ont mené dans une direction opposée où la frontière entre les deux actes est floue. Ce projet ethnographique étudie le vécu et les expériences des activistes du droit à la mort en Amérique du Nord, en Europe et en Australie, avec, entre autres, quatre mois de travail de recherche mené à Melbourne. Le but de cette étude visait à mieux comprendre comment la question a émergé de manière prépondérante au cœur des préoccupations politiques du $21^{\mathrm{e}}$ siècle et, plus particulièrement, comment les activistes du droit à la mort ont construit leurs revendications en se servant de leur propre vécu, mais aussi comment leur activisme politique a été reçu en fonction du vécu et des relations personnelles de chaque individu. Cette recherche a inclus l'analyse d'enquêtes législatives et l'observation participante sur le terrain lors d'activités de plusieurs

2. Par exemple, dans Norris v. Keith Bowers (2007). 
organismes défendant le droit à la mort, dont les réunions administratives, les événements entre membres, les discussions de groupes informelles et les ateliers d'information sur les manières de mettre fin à sa vie. J'ai aussi consulté les infolettres, publications, sites Web, enquêtes législatives, articles de journaux et témoignages publics pour analyser les arguments et le discours public sur la question. Soixante entrevues semi-dirigées ont été conduites auprès d'un vaste échantillon d'activistes et défenseurs de la cause, dont des médecins, des infirmières, des avocats, des figures politiques et d'autres individus associés au mouvement. Parmi eux, les présidents des organismes aussi bien que les membres de leur administration, les coordonnateurs et membres de manière plus générale. En ce qui concerne les organismes, la présente recherche a été menée auprès de groupes réformistes dominants s'investissant dans les réformes législatives ainsi qu'auprès de groupes plus radicaux prêchant une approche «DIY» (Do it yourself, ou «Faites-le vous-même») et offrant des renseignements sur la manière de mettre fin à sa propre vie au moment choisi.

Le présent article vise l'exploration du décalage existant entre un discours public qui sépare radicalement le suicide de la mort médicalement assistée et un discours privé qui confond ces deux gestes de mort volontaire. Bien qu'une opposition catégorique sépare apparemment ces deux discours, il reste des tensions et des ambigüités dans la vision du monde des activistes de la mort volontaire. Afin d'éclaircir le tout, je montrerai d'abord comment et pourquoi les deux gestes en sont venus à être séparés conceptuellement du point de vue de la rationalité, de l'intentionnalité et du choix. Puis, je montrerai comment la frontière entre le suicide et la mort médicalement assistée s'est estompée. Enfin, je tenterai de relier conceptuellement les deux gestes pour une compréhension plus critique de ce qui est en jeu. Pour ce faire, j'étudierai le débat qui persiste entre les activistes réformistes qui cherchent à faire changer la loi et les activistes plus radicaux agissant en dehors de celle-ci.

\section{La mort sanctionnée par la loi et la mort décriminalisée}

Une mort médicalement assistée se construit en opposition à un suicide même si les deux termes décrivent des gestes similaires; c'est-à-dire la prise intentionnelle de médication qui entraînera la mort. Cette mort médicalement assistée est un progrès récent: ce qui était communément 
appelé le «suicide assisté» dans la seconde moitié du $20^{\text {e }}$ siècle a été redéfini au siècle suivant, grâce aux activistes du droit à mourir, comme la «mort médicalement assistée». Le fondement logique qui pousse le mouvement activiste à séparer cet acte du suicide est clair. Il s'agit d'éviter ce qu'ils perçoivent comme une stigmatisation du suicide, mais aussi l'amalgame entre, d'un côté, la situation de ceux qui mettent fin à leur propre vie en raison de souffrances insupportables dues à des maladies dégénératives, intraitables ou en phase terminale et, de l'autre, toutes les autres formes de souffrance. Cette distinction est importante, car les opposants dans le débat soutiennent souvent que la légalisation de la mort assistée par un médecin légitimise l'acte du suicide et va jusqu'à en augmenter le nombre par une sorte de «contagion suicidaire» ${ }^{3}$. Pour eux, la distinction revient également à une simple tentative de «remplacement du vocabulaire» en vue de dissimuler ou d'obscurcir la problématique (Boudreau et Somerville, 2014, p. 7). De plus, en séparant conceptuellement les deux gestes en question, les activistes du droit à la mort cherchent à éviter l'écueil de la connotation négative associée au suicide qui tend à générer chez les membres des familles survivants une sorte de sentiment de honte. Par exemple, un billet du blogue de Dying With Dignity Canada (DWD Canada, Mourir dans la dignité Canada, 2016), intitulé «Pourquoi la mort médicalement assistée n'est pas un suicide [traduction libre]», explique que «le mot "suicide" est chargé d'une connotation négative et d'un bagage linguistique qui ne trouve pas sa place dans une conversation adulte et raisonnée sur le choix en fin de vie [emphase ajoutée]». De manière semblable, l'association américaine Death With Dignity propose une «terminologie de la mort assistée» où la différence est marquée entre les «termes corrects», comme "aide à mourir», «mort médicalement assistée», et les termes «suicide» et «suicide médicalement assisté». Du côté opposé, l'American Association of Suicidology (2017) a récemment déclaré que la mort médicalement assistée est reconnue comme «distincte du comportement traditionnellement appelé "suicide" ». Tout en restant neutre sur la question de la mort médicalement assistée, l'organisme soutient que la distinction élémentaire tient au fait que «le choix du patient n'est pas déformé par des troubles psychologiques».

3. Traduction libre de suicidal contagion: cet argument n'est soutenu par aucune donnée, comme c'est le cas pour l'argument principal contre la légalisation, à savoir que la mort assistée engendre invariablement des abus contre une population vulnérable. 


\section{Droit à la mort privé ou public}

Sur le plan conceptuel, une mort médicalement assistée, contrairement au suicide, sous-entend qu'un accord est donné avant de recevoir l'assistance en question. Les activistes du droit à mourir soulignent souvent ce qu'ils conçoivent comme une contradiction dans le cadre de la criminalisation de l'acte d'aider quelqu'un à mourir. D'une part, le droit de mettre fin à sa vie est reconnu implicitement dans la plupart des pays industrialisés où le suicide est, depuis longtemps, décriminalisé. Toutefois, là où des réformes législatives n'ont pas encore été mises en place, aider quelqu'un à mettre fin à sa vie est considéré comme un crime passible de peine de prison. Comment le fait d'aider quelqu'un à faire un geste légal peut-il être illégal? Dans les faits, il est reconnu depuis longtemps que les «homicides par compassion» sont rarement poursuivis et mènent encore plus rarement à des condamnations en raison de leurs circonstances atténuantes, surtout si les motivations de l'individu faisant le geste d'assistance ne sont pas remises en question. D'autre part, une mort médicalement assistée est un acte sanctionné par la loi qui fait appel à un professionnel, supposé désintéressé, du milieu médical et travaillant dans le cadre de ses capacités professionnelles. L'individu mettant fin à sa vie d'une telle manière doit répondre à des critères d'admissibilité médicale. Ces critères peuvent se définir strictement et dans le cadre de maladies en phase terminale qui laissent raisonnablement prévoir la mort naturelle dans une période de six mois à un an, ce qui comprend de manière large les cancers avancés (selon les modèles de l'Oregon et de Washington). Ils peuvent aussi être définis plus généralement en fonction de souffrances insupportables dans le contexte de diagnostics médicaux (selon les modèles de la Belgique et des Pays-Bas). Ajoutons que l'accès à l'exercice de ce droit implique des examens médicaux, plusieurs formulaires de consentement, des témoins, des périodes d'attente et autres procédures administratives qui s'assurent à la fois du diagnostic médical et, surtout, du souhait persistant de mourir basé sur la décision «libre» (c.-à-d. non contrainte) de l'individu concerné.

Selon Foucault (1976/1990), dans Histoire de la sexualité I, La volonté de savoir, le suicide relève du «droit de mort» privé depuis que le suicide n'est plus un crime contre le souverain. En Angleterre, la peine pour ce crime pouvait conduire autrefois à la confiscation de tout héritage pour la famille ou encore à l'enterrement du mort à la croisée de chemins; en France aussi, la condamnation pouvait être une confiscation des biens 
et la profanation du corps. Avec l'émergence de la biopolitique, l'individu est devenu libre de commettre un suicide (c'est-à-dire que ce n'était plus un acte condamnable). Le pouvoir souverain a ainsi resserré ses efforts pour exercer un contrôle sur la vie en tentant de prévenir et de pathologiser le suicide. Ce geste est donc devenu un élément central des connaissances émergentes en sociologie et en psychologie ainsi qu'un objet grandissant d'intervention. À travers ces changements, le suicide est devenu un geste pathologique et irrationnel, un produit de troubles psychologiques qui doit être prévenu (Marsh, 2010). Il n'est dès lors plus un acte «raisonné» ou «politique».

Si le suicide est un droit de mort privé, la mort médicalement assistée est un droit de mort public qui pose donc un problème plus direct à l'insistance biopolitique du maintien de la vie. Lactivisme du droit à mourir existe dans un espace où, comme le constate l'anthropologue Sharon Kaufman (2015), les technologies médicales et les systèmes de santé ont tendance à imposer des interventions médicales et des traitements qui peuvent prolonger la vie, mais, souvent, dans de grandes souffrances. Le droit à la mort est une intervention médicale qui remet en question l'aspect dominant des dites interventions. Si le suicide est plutôt conçu comme le geste irrationnel d'un sujet irrationnel, une mort médicalement assistée se fonde d'abord sur le geste raisonné d'un individu rationnel. Cette distinction est un point polarisant du mouvement du droit à mourir dans sa quête de réformes législatives. La rationalité, le raisonnement de l'individu concerné et l'intelligibilité de ses motivations deviennent dès lors les principes décisifs dans la mort assistée. En définissant le souhait de mettre fin à sa propre vie dans un contexte manifestement rationnel, l'action devient légitime et peut être sanctionnée par la loi, et donc assistée par un professionnel. Cet éclaircissement fait toute la différence entre une mort médicalement assistée et un suicide.

\section{La liberté devant la contrainte}

La mort médicalement assistée en tant que résultat d'un processus décisionnel raisonné se base donc sur le choix. L'infrastructure judiciaire qui entoure une mort assistée se concentre sur le caractère volontaire de ce choix. La demande de mettre fin à sa vie et le consentement informé de l'individu concerné doivent être documentés et soumis à l'approbation de tierces personnes. Dans une logique semblable, les 
terminologies utilisées par le passé font la différence entre l'euthanasie «volontaire» et «involontaire», distinction où «l'euthanasie involontaire» (c.-à-d. le meurtre) faisait référence à un système de croyances nazi comprenant le concept de Lebensunwertes Leben (vie indigne de vie). Une mort volontaire faisait tout simplement référence au fait que l'individu n'était pas victime d'un meurtre. L'amalgame ou le glissement entre ces deux termes par les opposants de la légalisation de la mort assistée (amalgame que mes sources compareraient avec exaspération au fait de ne pas pouvoir discerner un rapport sexuel entre adultes consentants d'un viol) est la raison pour laquelle le terme «euthanasie» a été abandonné presque complètement, sauf par les opposants de la légalisation (voir Michalsen et Reinhart, 2006). Les activistes construisent leurs campagnes autour de la notion de choix, plus précisément le droit d'avoir le choix de mourir au moment et de la manière choisis. L'idée se résume ainsi : «ma mort, mon choix», ou à chacun son «choix de fin de vie».

Toutefois, si le caractère volontaire du geste est primordial, on peut en effet déduire que les individus demandant la mort assistée souhaitent en effet mettre fin à leur vie. Mais malgré cette importance de la volonté dans le droit à la mort, une mort médicalement assistée n'est pas conçue comme un acte de volonté, fait qui joue aussi un rôle central dans la différenciation de ce geste et du suicide. Commettre un suicide revient à s'enlever la vie lorsque d'autres options (supposées meilleures) existent. Mettre fin à sa propre vie par assistance médicale est un choix de dernier recours lorsqu'aucune autre option ne reste. Si le geste est conçu nécessairement comme un choix «libre», ce choix existe dans un contexte surdéterminé et est précipité par une cause médicale qui le contraint. Parce que le sujet vit une souffrance décrite comme intolérable ou insupportable, la seule volonté qui lui est encore laissée est celle de se voir contraint de s'enlever la vie alors que, dans d'autres circonstances, il ne le ferait pas. Il ne choisit pas de mourir. Il choisit simplement comment mourir. Comme l'explique un activiste du droit à mourir: "C'est comme prendre une pilule de cyanure quand l'ennemi est sur le point de vous capturer, et que s'il vous capture, vous savez qu'il vous torturera pendant des mois avant de vous tuer de la manière la plus douloureuse possible.» Un autre encore parle d'un choix fait sous la menace d'une arme. Cath, elle aussi activiste du droit à la mort et souffrant d'une leucémie au stade 4 et d'un cancer du sein, m'explique qu'elle n'aime pas le terme suicide parce que «c'est quand on détruit sa 
propre vie pour des raisons tordues. Ici, c'est différent. Ici, ce n'est pas un choix. Je ne le vois pas comme un suicide. Je choisis de ne pas mourir d'une mort lente et douloureuse. C'est le seul choix. La mort est inévitable».

Le protagoniste du documentaire The Suicide Tourist, Craig, un professeur américain à la retraite qui souffre de sclérose latérale amyotrophique (SLA) et qui a choisi de mettre fin à sa vie en allant en Suisse, explique: «ce ne serait pas mon choix si j'avais d'autres options». Craig souligne: «C'est la mort ou la souffrance et la mort.» Ainsi, les activistes soutiennent que la cause de décès de patients souffrant de maladies comme la SLA et choisissant de mettre fin à leur vie ne devrait pas être le suicide, mais la maladie elle-même. Craig n'a pas choisi de mettre fin à sa vie; la SLA, qui l'aurait tué autrement, l'a contraint à ce choix. Considérant l'accent mis sur le choix dans les campagnes publiques du mouvement du droit à mourir, ce rejet du choix dans les entrevues est révélateur. Bien que le choix soit le fondement de l'activisme du droit à la mort, il ne s'agit pas d'un choix entre la vie et la mort, mais d'un choix entre des morts: une "bonne mort» (brève et sans douleur) et une «mauvaise mort» (longue, fastidieuse, douloureuse, qui se déroule dans la souffrance, l'anxiété ou la perte de contrôle sur son propre corps). Le suicide, au contraire, est construit comme un choix entre la vie et la mort.

Ce manque de choix dans la mort assistée est ce qui rend le choix en question rationnel. Alors que l'on considère le choix du suicide comme un "mauvais choix», un individu qui meurt avec l'assistance d'un médecin veut échapper à une souffrance prolongée et invalidante, veut éviter une «mauvaise mort», veut éviter de voir sa famille souffrir d'être témoin de ses propres souffrances. D'un autre côté, le suicide est un «mauvais choix» puisque fondé, dans les mots de Cath, sur des raisons «tordues» (qui découlent, on présume, de troubles psychologiques). Mais faire cette distinction peut amener certains activistes du droit à la mort à une attitude désinvolte relativement au suicide. Par exemple, un député canadien défenseur de la cause qui a soumis plusieurs projets de loi d'initiative parlementaire pour la légalisation de la mort médicalement assistée, a déclaré en entrevue: «Le suicide est un terme chargé. C'est pourquoi je parle de mort médicalement assistée. Je ne parle pas d'adolescents qui se lèvent du mauvais pied.» Paul, un activiste qui faisait partie, dans les années 1990, de ce qu'on appelait «l'euthanasie clandestine» en Amérique du Nord, réseau qui fournissait de 
l'assistance illégalement à des gens qui souhaitaient mettre fin à leur vie, se rappelle «avoir passé l'après-midi avec quelqu'un qui s'est révélé être un cinglé. Cette personne voyait tout ça comme un geste romantique. C'était une jeune femme qui souffrait de dépression... pas vraiment rationnelle.» Pour sa part, Jay, activiste et gestionnaire des réseaux sociaux pour le droit à la mort, souffre d'une maladie chronique qui le laisse constamment dans d'atroces douleurs. Il a publié le lien d'un article expliquant la différence «entre avoir des tendances suicidaires et vouloir mourir ». Dans ses mots, le premier cas relève de «l'émotion» alors que souhaiter mourir se fonde sur un «acte rationnel reposant sur la considération de la situation présente et future». Ainsi, la mort médicalement assistée découle d'une dichotomie corps/esprit: un «esprit sain» dans un «corps malade», ce qui s'oppose au suicide qui découle d'un «esprit malade» dans un «corps sain».

\section{L'inévitabilité de la mort}

Les adolescents émotifs «qui se lèvent du mauvais pied» et les «cinglés» irrationnels qui voient le suicide sous un angle romantique et mettent fin à leur vie avec désinvolture sont donc mis en parallèle avec des individus qui le font dans un processus rationnel, réfléchi et délibéré en contexte de conditions médicales terminales, dégénératives ou incurables. Cette dynamique à présent établie en tant que dichotomie centrale déchirant le milieu activiste du droit à la mort, il me reste à explorer les ambiguiités, ambivalences et contradictions qui existent dans cette vision des choses, qui en appelle à une réalité plus floue et plus nuancée. Si le suicide est, comme Cath le comprend, «détruire sa propre vie pour des raisons tordues», qui en juge concrètement? Ceci pose surtout un problème puisque presque tous les activistes du droit de mourir reconnaissent que la "souffrance intolérable», le critère primordial dans le souhait de mettre fin à sa propre vie pour la plupart d'entre eux, est un phénomène subjectif; bref, un phénomène qui dépend de l'expérience individuelle, qui est «profondément personnel» et qu'il est donc difficile d'évaluer (Dees, Vernooij-Dassen, Dekkers et van Weel, 2010). Plus encore, la douleur et la souffrance sont aussi reconnues comme des éléments qui influencent le jugement. Qui peut juger de la douleur et de la souffrance de quelqu'un d'autre? Comme Elaine Scarry (1987) l'écrit, «la douleur appartient à ceux qui la vivent; et quand d'autres décident à leur place de ce qu'elle est pour eux, les débordements ne sont jamais loin [traduction libre]» (p. 4). 
En fait, les activistes du droit de mourir témoignent d'une large variabilité concernant l'expérience individuelle de la souffrance et rappellent que la souffrance intolérable pour l'un peut être tolérable pour un autre. Plus encore, ils soutiennent que la souffrance ne devrait pas être comprise comme la manifestation physique d'une douleur causée directement par une maladie. Elle ne devrait plus être mesurée (inadéquatement) par des échelles et être si souvent ignorée par le système médical. Ces activistes dirigent plutôt les débats vers la souffrance psychologique et existentielle comme critère prépondérant pour cadrer et déterminer le désir de mourir. Ces faits sont aussi soutenus par tous les travaux empiriques sur les raisons pour lesquelles les gens mettent fin à leur vie par assistance médicale (Monteforte-Royo, Villavicencio-Chávez, Tomás-Sábado, Mahtani-Chugani et Balaguer, 2012; Pearlman et al., 2005). Cette recherche dévoile que les raisons existentielles et psychologiques sont plus importantes que la douleur physique dans les requêtes de mort assistée. Si la souffrance physique est déjà difficile à mesurer, la souffrance existentielle est encore bien davantage un problème pour les professionnels de la santé. Comme Richards (2017) le conclut dans son étude ethnographique des «touristes britanniques du suicide» en Suisse: «la souffrance est rarement somatique, elle est plutôt attachée aux vécus, aux sphères sociales et aux dilemmes existentiels des gens [traduction libre]» (p. 359).

La distinction entre la souffrance physique et la souffrance psychologique ou existentielle ouvre un débat encore plus épineux. L'aspect rationnel à l'origine du souhait de mettre fin à sa vie est façonné par une dichotomie esprit/corps qui construit un choix entre un «esprit sain» (capable de faire un choix raisonné) et un «corps malade» (l'état de santé qui contraint ce choix). Cette dichotomie a été longtemps critiquée et est reconnue comme un problème dans la pratique biomédicale (Damasio, 1994; Gallagher, 2006), particulièrement par les anthropologues (Scheper-Hughes et Lock, 1987). En termes pratiques, cela soulève la question des personnes souffrant de troubles psychologiques et de leur admissibilité à la mort assistée. La plupart des activistes du droit de mourir considèrent les problèmes de santé mentale comme une catégorie problématique, même si plusieurs seraient d'accord pour considérer comme également admissibles les personnes souffrant grandement de problèmes de santé mentale incurables. Et ils posent la question suivante: pourquoi la souffrance intolérable causée par un trouble mental insurmontable devrait-elle 
être perçue différemment de la souffrance intolérable causée par une maladie physique insurmontable?

De telles réflexions ouvrent également le débat, pour les activistes, de la séparation de la souffrance physique des autres formes de souffrances. Cath, par exemple, est une activiste qui souscrit à l'idée que les personnes âgées «fatiguées de vivre» devraient aussi avoir la liberté de mettre fin à leur vie puisque leur mort (probablement de vieillesse) est aussi «inévitable». Dans ces cas, la motivation de mettre fin à une vie est existentielle et se rapproche plus d'une forme «d'ennui» que d'un cas extrême de souffrance psychologique. Mais la frontière entre suicide et mort médicalement assistée devient plus floue encore lorsqu'on se penche sur l'idée de "l'inévitable». Cath finit par me parler d'un «vrai suicide» dans sa vie, celui de son premier mari, qui souffrait selon elle de dépression, de maladie mentale et de toxicomanie, événement qu'elle différencie de la manière rationnelle de mettre fin à sa vie. Elle conclut: «Il ne pouvait pas recevoir de traitement. [...] Rien de ce qu'ils faisaient pour lui ne pouvait l'aider, le trouble dont il souffrait était dans sa phase terminale.» Ainsi, sa mort, le résultat d'un «trouble en phase terminale», était, elle aussi, «inévitable». En d'autres mots, ce qu'elle considère comme un «vrai» suicide provoqué par des troubles mentaux répond aux mêmes critères d'«inévitabilité» qu'elle attribue à la mort médicalement assistée et provoquée par une maladie en phase terminale. Quant à Jessica, la description qu'elle fait du suicide de son père au début de cet article est similaire. Elle m'explique: «C'était un choc, mais je défendrais encore son droit à le faire.» Lorsque je lui demande de préciser, elle me répond: "C'était son choix et je le respecte.» Lorsque je lui demande si elle pense que sa décision était «raisonnée», elle explique: "pas nécessairement, mais il n'y a aucune garantie que les choses auraient été mieux s'il ne l'avait pas fait. Il ne voyait aucune autre issue et, oui, c'était triste. C'était triste qu'il soit rendu là, mais c'était son corps, son choix».

\section{«Tant que les quincailleries vendront de la corde, il y aura des suicides $»$}

Malgré la dichotomie entre le suicide et la mort assistée, les suicides peuvent et devraient être prévenus. Une mort assistée ne peut l'être, car elle survient dans un contexte où la mort est conçue comme inévitable. Mais comme nous venons de le voir, même cette dichotomie ne peut 
pas toujours être maintenue. Les activistes du droit de mourir sont en effet critiques de ce qu'ils perçoivent comme le «mythe» de la prévention du suicide. Comme Jessica l'explique, «tant que les quincailleries vendront de la corde, il y aura des suicides». Des années après le suicide de son mari, Binna, dont l'histoire est en ouverture de l'article, a suivi un cours de formation continue sur le suicide. Elle raconte qu'une des questions posées par l'enseignant était de savoir si les suicides devraient toujours être prévenus. Alors que la classe soutenait de façon quasi unanime cette idée, Binna dit qu'elle ne croyait pas que tous les suicides pouvaient ou même devraient moralement être empêchés «parce qu'on ne sait pas de quoi il s'agit». En réfléchissant à la situation de son mari, elle voit son suicide comme surdéterminé. Il n'aurait pas pu être empêché. Elle se demande même si ce geste déclenché par des événements dans sa vie n'était pas lié à une expérience vécue dans sa petite enfance.

Cette certitude pourrait l'aider à soulager toute culpabilité qu'elle puisse sentir relativement à la mort de son mari. Si son suicide était inévitable, rien qu'elle aurait pu faire ne l'aurait aidé. Lactivisme qui survient plus tard dans la vie de Binna semble évoquer que, même si le suicide de son mari n'avait pas pu être empêché, peut-être qu'une manière moins violente de mettre fin à sa vie aurait été possible. Comme Binna le déplore plus tard dans notre discussion, une des questions auxquelles elle a songé renvoyait à «une manière plus civilisée de faire les choses». Son point de vue est très compréhensible compte tenu du traumatisme que le suicide de son mari a dû avoir sur elle en tant que témoin. Cette mort violente et sanglante doit se juxtaposer dans son esprit à une mort assistée, sous narcotiques pharmaceutiques, celle qu'elle défend: une mort «en paix» où l'individu semble être mort dans son sommeil lorsqu'on retrouve son corps. De la même façon, les suicides violents et le traumatisme qu'ils provoquent chez les autres ont forgé la vision du monde de plusieurs de mes informateurs. Johannes, coordonnateur national d'un organisme pour le droit de mourir, voit son intérêt professionnel pour la mort remonter au suicide d'un de ses amis par auto-immolation. Lindy, coordonnatrice régionale d'un organisme similaire parle du suicide du père de son petit ami lorsqu'elle était adolescente. Elle était chez eux au moment où toute la famille a entendu le coup de feu qui a mis fin à sa vie. Jessica parle pour sa part du suicide de son oncle, au Royaume-Uni, qui s'est tué en se jetant devant un train. Johannes raconte à l'une des rencontres de groupe: «Je préfère que mon fils se tue au Nembutal (un narcotique pharmaceutique que son orga- 
nisme pro mort assistée promeut comme méthode) plutôt qu'en sautant devant un train.» Durant notre entretien, il ajoute: «La réalité, c'est que des gens se suicident tous les jours de toutes sortes de manières horribles, violentes et traumatisantes. Ce n'est pas traumatisant que pour eux, mais pour leur famille, pour le personnel des urgences, les chefs de trains ou la police. C'est horrible pour tout le monde.»

\section{Les franges radicales de l'activisme du droit de mourir}

L'activisme du droit à la mort, comme bien d'autres mouvements sociaux, peut être divisé grossièrement en deux positions: les radicaux et les réformistes. Les réformistes travaillent au sein du système afin de pousser à des réformes législatives. Les lois sur le droit à la mort comprennent des critères d'admissibilité et de nombreux garde-fous contre des abus potentiels. Les radicaux insistent sur l'insuffisance de la loi parce que les critères d'admissibilité excluent forcément certains individus qui en appellent de ce droit. Ils soutiennent également que les garde-fous, que ce soit les périodes d'attente et les consultations médicales, peuvent en outre devenir des sources de souffrance. Quand les réformistes montrent du doigt des lois qui fonctionneraient en raison du pourcentage des gens demandant l'aide à la mort dont la requête est refusée (par exemple, une étude de Snijdewind, Willems, Deliens, Ouwuteaka-Philipsen et Chambaere [2015] aurait montré que près de la moitié des requêtes sont refusées dans une clinique des Pays-Bas), les radicaux parlent plutôt des limites de ces mêmes lois. La position radicale se tient en fait au-delà de la loi et était appelée, il y a quelques années, «l'euthanasie clandestine» (euthanasia underground) (Magnusson, 2000). Ses activistes défendent l'approche «Faites-le vous-même» (DIY). Ils créent donc des réseaux de soutien et échangent des renseignements sur des manières fiables de mettre fin à sa propre vie pour autonomiser les individus qui décident de prendre eux-mêmes les choses en main plutôt que de dépendre du système médical. En agissant en dehors ou en marge de la loi, les membres de tels organismes radicaux peuvent se heurter à des conséquences judiciaires.

Des participants de tout horizon de l'activisme du droit de mourir ont été recrutés dans le cadre de ma recherche ethnographique et plusieurs fils conducteurs existent entre leurs positions. Certains d'entre eux faisaient partie d'organismes à la fois réformistes et radicaux, 
défendant tant l'aspect pragmatique les poussant à soutenir les réformes législatives que les limites de ces dernières, surtout là où aucune loi sur le droit de mourir ne s'applique, d'où leur implication dans des options hors du cadre législatif. Par exemple, des participantes de cette étude, Cath et Binna, sont membres d'Exit International, un organisme qui se tient du côté le plus radical de la problématique du «suicide rationnel», c'est-à-dire de l'idée que toute personne ayant un «esprit sain» devrait avoir le droit de mettre fin à sa propre vie indépendamment de tout diagnostic médical. Exit est né en Australie, mais est devenu un organisme international dont des sections régionales actives se sont étendues au Royaume-Uni, en Nouvelle-Zélande, aux États-Unis et au Canada. Ils soutiennent que le droit à la mort ne devrait pas être sous le contrôle des professionnels de la santé pour éviter un «paternalisme médical» et la «médicalisation» d'une question sociale. Cette critique provient de l'expérience et de la philosophie du fondateur de l'organisme, Philip Nitschke. Son propre activisme découle de son expérience en tant que professionnel de la santé dans le Territoire du Nord, en Australie, premier endroit au monde à légaliser la mort assistée en 1996, avant de voir le Parlement du pays annuler la loi quelque mois après. Durant cette courte période, Nitschke était le seul médecin qui se servait de cette loi, ayant aidé trois individus souffrant de maladies en phase terminale à mettre fin à leur vie. Une fois la loi nulle, Nitschke devint activiste de la méthode DIY par deux moyens principaux: soit en se procurant du Nembutal, un pentobarbital sous prescription (qu'il est possible d'acheter en ligne en Chine ou en personne dans des banques d'approvisionnement vétérinaire au Mexique) ou par cartouches de nitrogène (offertes légalement pour servir de la bière pression). Les critères d'admission de l'organisme sont d'être âgé de plus de 50 ans et de n'avoir aucun antécédent de maladie mentale. Nitschke admet que ces deux critères sont arbitraires, mais il les considère comme nécessaires devant l'attention judiciaire que l'organisme a attirée; attention qui est allée jusqu'à l'arrestation de plusieurs membres. Nitschke a lui-même vu son domicile perquisitionné, a été interrogé à de nombreuses reprises et s'est vu retirer son permis d'exercice (pour le récupérer seulement après une lutte judiciaire et puis le brûler) pour ne pas avoir dirigé un membre vers de l'aide psychiatrique, membre qui a fini par se suicider. Il n'était pas en mauvaise santé mais était suspecté d'avoir tué sa femme et une ancienne petite amie. Exit est controversé même parmi les organismes de droit à la mort les plus connus. Plusieurs 
l'accusent d'être «irresponsable», précisément à cause de sa position plus ouverte et sans "garde-fous médicaux». En diffusant des renseignements sur les manières de mettre fin à sa vie et en tenant un site Web où figure un forum qui permet d'échanger ouvertement des renseignements et des idées, l'organisme a été accusé à répétition par des opposants, des médias, des politiciens et même d'autres activistes de faire du death coaching ou encore de rendre un «culte à la mort» qui encouragerait les gens à se suicider.

En Australie et au Royaume-Uni, on blâme Exit pour le suicide de plusieurs jeunes. Un exemple flagrant est celui de Lucas, un jeune Australien de 26 ans résidant à Berlin. En prétendant être âgé de 65 ans, il s'enregistre en tant que membre d'Exit pour recevoir l'accès à leur manuel, à leur site internet et aux forums de discussion. Il finit par utiliser la méthode recommandée et met fin à sa vie par une surdose de Nembutal importé illégalement. La mère de Lucas a attribué ouvertement le blâme à l'organisme en expliquant qu'elle ne pouvait comprendre pourquoi son fils se serait suicidé puisqu'il ne paraissait ni dépressif ni suicidaire. La seule explication est pour elle qu'Exit en était responsable et, si les renseignements en question n'avaient pas été mis à sa disposition, il serait encore en vie. Contrairement aux membres d'Exit qui voient le suicide de leurs proches comme «inévitable», la mère de Lucas croit plutôt que le suicide de son fils aurait pu être empêché. D'une certaine manière, Lucas s'est vu retirer tout pouvoir sur son suicide. Son acte ne semble pas être le produit de sa volonté, mais plutôt le résultat des actions de tiers. Lors d'une réunion à laquelle j'ai assisté, Johannes, ancien coordinateur national d'Exit, parle de la mort de Lucas aux autres membres: «nous sommes nous aussi bouleversés par ce qui est arrivé à ce jeune», soulignant le fait qu'il y a des personnes vivant des situations «tragiques, qui sont désespérées et qui ont besoin d'aide». Lors de notre entretien, il m'explique que la vaste majorité des gens qui mettent fin à leur vie souffrent «sans aucun doute» de maladies mentales, "mais il y en a beaucoup pour qui ce n'est pas le cas. Il existe des processus différents qui sont dans un sens rationnels». Comme il l'exprime aux autres membres du groupe, «le suicide est la première cause de mortalité chez les jeunes dans la plupart des pays industrialisés riches. Un million de personnes mettent fin à leur vie tous les ans. Quatre-vingt-dix-neuf pour cent des cas sont tragiques. Nous nous battons pour le un pour cent qui reste». Toutefois, Johannes, comme d'autres activistes, se réfugie rapidement dans la 
dichotomie entre les deux actes étudiés ici en concluant: «nous avons besoin d'un mot différent pour ces personnes qui mettent fin à leur vie de manière rationnelle».

En parallèle, en entrevue avec nous, Nitschke adopte une vision englobante et ouverte de ce qu'«être sain» voudrait dire:

Déterminer si oui ou non une personne est capable, peu importe ce qu'on veut dire par là et je suis conscient que c'est très vague, c'est reconnaître qu'au sens de la loi qui existe présentement, si vous êtes considéré comme un danger pour vous-même ou pour les autres, vous pouvez être placés obligatoirement dans un établissement psychiatrique. J'aurais tendance à dire que, si vous n'êtes pas dans tel établissement et que vous n'êtes pas entouré de gens qui cherchent à déterminer si vous devriez l'être, vous êtes capable. Vous prenez vos propres décisions sur votre vie. Certains ne seraient peut-être pas d'accord avec votre décision, plusieurs même ne le seront pas, mais l'État n'a pas son mot à dire, d'après moi, si vous choisissez de mettre fin à votre vie. (traduction libre)

Ainsi, un «esprit sain», un peu comme la responsabilité criminelle, permet peu d'exceptions depuis la désinstitutionnalisation et, de nos jours, peu de gens souffrant de maladies mentales sont institutionnalisés contre leur volonté (Sealy et Whitehead, 2004). Pour Nitschke, «être capable», c'est n'être pas considéré comme «fou» (et constitue plus un jugement légal que psychiatrique). Sa position prend en compte le fait que la plupart des personnes ayant des problèmes de santé mentale diagnostiqués sont elles aussi capables. Nitschke est lui-même influencé par le mouvement antipsychiatrie, surtout par les travaux du libertarien Thomas Szasz, qui a défendu quelques années avant Nitschke une position controversée contre les principes de la prévention du suicide (Szasz, 1971). Mais les activistes du droit de mourir ne partagent pas tous cette vision des choses et nombreux sont ceux à s'en distancier. Toutefois, selon mes recherches, je conclurais que les convictions personnelles de la majorité de ces activistes se rangent plutôt du côté des grandes catégories du suicide rationnel que de l'accent mis sur les maladies en phase terminale qui laissent six mois à vivre aux patients. En fait, cet accent mis sur les maladies en phase terminale (comme les modèles de l'Oregon et de Washington) était perçu comme trop restrictif par tous les activistes interrogés dans le cadre de ce projet de recherche.

La critique de Nitschke laisse place à une ambiguiité et à une tension centrale : bien que la souffrance puisse être d'origine physique, souffrir 
ne peut pas être médicalement défini ou compris. Pour Nitschke, la souffrance d'origine médicale ne devrait pas être une catégorie séparée des souffrances d'autres origines, qu'elles soient romantiques, sociales, économiques, politiques ou autres. Selon la vision de Nitschke, une telle séparation est impossible à justifier du point de vue philosophique, et n'est pas nécessaire en pratique puisque chacun devrait pouvoir exercer son droit sans assistance médicale. Ainsi, son organisme promeut le droit absolu d'avoir accès aux renseignements les plus fiables sur les manières de mettre fin à sa propre vie (ainsi qu'aux moyens nécessaires). Sa vision se situe moins en fonction de la loi qu'en dehors de celle-ci; il rejette les réformes légales pour une décriminalisation radicale. Il remet plutôt en question qu'un droit de mourir puisse exister s'il est restreint à un nombre limité d'individus sous une batterie de conditions définies par des tiers et sujettes à des vérifications médicales, de la supervision et des approbations. Dans ses mots:

Au cours des vingt dernières années, j'ai rencontré beaucoup de gens qui avaient des raisons valables d'avoir recours à la législation en place, mais sans jamais répondre aux critères établis pour y accéder. Un certain nombre d'entre eux avaient des raisons sociales de vouloir mourir. [Les organismes réformistes du droit de mourir] soutiennent que vous avez le droit de mourir, mais elles essaient de restreindre ce droit. C'est incohérent. Soit vous avez le droit, soit vous ne l'avez pas! (traduction libre)

\section{Conclusion: "Les gens ne veulent pas mourir»}

Le présent article souligne des problématiques conceptuelles qui sont au cœur de la mort médicalement assistée. Que signifie la mise à l'écart d'une façon particulière de mettre fin à sa vie? La vision des activistes radicaux du droit de mourir remet en question la ligne qui sépare la mort médicalement assistée, causée par les souffrances insoutenables d'une maladie physique, et le suicide irrationnel, causé supposément par un trouble psychologique. Leur critique rappelle la position de Jean Améry, survivant de l'Holocauste et essayiste, dans son livre Du suicide (1999). Améry s'opposait aux psychologues et suicidologues en rejetant l'idée qu'une «mort volontaire» pourrait ou devrait être comprise à partir d'un point de vue externe ou par un jugement qui peut considérer une chose rationnelle et une autre, absurde ou arbitraire. Il insiste plutôt sur la dimension phénoménologique de la souffrance, de l'humiliation, de la déception, de l'angoisse et de l'échec qui poussent les 
individus à mettre fin à leur vie. Plus encore, il soutient que la frontière entre la mort volontaire et la mort involontaire est elle-même poreuse. Les gens peuvent «se tuer» de plusieurs manières, par exemple par la drogue, l'alcool, la cigarette, en prenant part à des activités dangereuses, etc. En comprenant ce qui pousse quelqu'un à une mort volontaire, Améry veut montrer que les êtres humains sont pris dans une lutte entre une logique de vie animée par le désir de vivre et une logique de mort (ou antilogique, puisque la mort défie toute logique au sens existentiel - et sa conception se distingue d'une certaine façon de la pulsion de mort de Freud) guidée par un désir de mourir.

Cette idée peut aider à exposer un des paradoxes les plus intéressants de l'activisme du droit à la mort, qui est sous-jacent aux idées préconçues du milieu sur la mort assistée. Plutôt que d'être guidés par une logique de mort, ces activistes suivent une logique de vie. Même en se battant pour le droit des gens à mourir, leur croyance principale est que le désir de vivre est bien plus fort que le désir de mourir. En se demandant si sa vision rend «trop facile» le geste de mettre fin à sa vie, Nitschke me dit qu'il rejette l'idée que «la seule raison d'être en vie est de ne pas encore savoir comment mourir». Mais plus fondamentalement, il assure que «les gens ne veulent pas mourir... ils veulent cette drogue, mais ne veulent pas la prendre. Ils ne veulent que savoir qu'elle est là. Ils veulent qu'elle reste une option ». Nitschke croit fermement que si le Nembutal était offert à tout adulte (voire même sans prescription), les taux de suicide n'augmenteraient pas.

Au bout du compte, les activistes du droit de mourir remettent en question le fait que le souhait de mourir soit intrinsèquement conçu comme étant pathologique. Comme Cath, ancienne infirmière au dernier stade d'une leucémie et d'un cancer du sein, me le dit: «les gens en santé mentale ici croient tous que le suicide, le désir de se suicider, est un sentiment anormal qui doit être traité et arrêté». Cette perspective rappelle l'homonyme de Nitschke, Nietzsche, qui écrivait: «La pensée du suicide est une puissante consolation, elle aide à passer plus d'une mauvaise nuit.» En d'autres mots, pour ces activistes, avoir accès aux moyens de mettre fin à sa vie et en avoir les capacités peut augmenter le bien-être et aider les gens, contre-intuitivement, à vivre plus longtemps dans des circonstances incroyablement difficiles. Les activistes du droit de mourir ont peut-être en commun le fait de comprendre que le désir de mourir peut être la seule manière, pour certains, de trouver le désir de vivre. 


\section{Références}

American Association of Suicidology. (2017). "Suicide" is not the same as "physician aid-in-dying”. Repéré à http://www.suicidology.org/Portals/14/docs/ Press \% 20Release/PAD \% 20Statment \% 20Release.pdf? ver=2017-11-05$165657-723$

Améry, J. (1999). On suicide: a discourse on voluntary death. Bloomington, IN: Indiana University Press.

Boudreau, J. D. et Somerville, M. (2014). Euthanasia and assisted suicide: a physician's and ethicist's perspective. Medicolegal and Bioethics, 4, 1-12.

Damasio, A. (1994). Descartes' error: Emotion, reason and the human brain. New York, NY: Harper Perennial.

Dees, M., Vernooij-Dassen, M., Dekkers, W. et van Weel, C. (2010). Unbearable suffering of patients with a request for euthanasia or physician-assisted suicide: an integrative review. Psycho-Oncology, 19, 339-352.

Dying With Dignity Canada. (2016, 23 septembre). Why medically assisted dying is not suicide [Billet de blogue]. Repéré à http://www.dyingwithdignity. ca/assisted_dying_is_not_suicide

Foucault, M. (1990). The history of sexuality, vol. 1 : An introduction (traduit par R. Hurley). New York, NY: Vintage Books. (Ouvrage original publié en 1976 sous le titre Histoire de la sexualité I, La volonté de savoir).

Gallagher, S. (2006). How the body shapes the mind. Oxford, Royaume-Uni: Oxford University Press.

Kaufman, S. (2015). Ordinary medicine: Extraordinary treatments, longer lives, and where to draw the line. Durham, NC: Duke University Press.

Magnusson, R. (2000). Angels of death. Exploring the euthanasia underground. New Haven, CT: Yale University Press.

Marsh, I. (2010). Suicide: Foucault, history and truth. Cambridge, Royaume-Uni: Cambridge University Press.

Michalsen, A. et Reinhart, K. (2006). "Euthanasia”: A confusing term, abused under the Nazi regime and misused in present end-of-life debate. Intensive Care Medicine, 32(9), 1304-1310.

Monteforte-Royo, C., Villavicencio-Chávez, C., Tomás-Sábado, J., MahtaniChugani, V. et Balaguer A. (2012). What lies beneath the wish to hasten death? A systematic review and meta-ethnography from the perspective of patients. PLoS One, 7(5), e37117.

Pearlman, R. A., Hsu, C., Starks, H., Back, A. L., Gordon, J., Bharucha, A. J., ... Battin, M. P. (2005). Motivations for physician-assisted suicide. Journal of General Internal Medicine, 20(3), 234-239.

Richards, N. (2017). Assisted suicide as remedy for suffering? The end-of-life preferences of British "suicide tourists". Medical Anthropology, 36(4), 348-362.

Scarry, E. (1987). The body in pain: The making and unmaking of the world. New York, NY: Oxford University Press.

Scheper-Hughes, N. et Lock, M. (1987). The mindful body: A prolegomenon to future work in medical anthropology. Medical Antbropology Quarterly, 1(1), 6-41. 
Sealy, P. et Whitehead, P. (2004). Forty years of deinstitutionalization of psychiatric services in Canada: An empirical assessment. Canadian Journal of Psychiatry, 49(4), 249-257.

Snijdewind M. C., Willems D. L., Deliens, L., Onwuteaka-Philipsen, B. D. et Chambaere, K. A. (2015). Study of the first year of the end-of-life clinic for physician-Assisted dying in the Netherlands. JAMA Intern Med, 175(10), 1633-1640.

Szasz, T. (1971). The ethics of suicide. The Antioch Review, 31(1), 7-17.

\title{
Jurisprudence
}

Norris v. Keith Bowers. (2007, 9 août). Repéré à http://caselaw.findlaw.com/ us-8th-circuit/1453877.html

\section{"99\% of suicides are tragic; we're fighting for the other $1 \%$ ": "sound minds" and unsound bodies in right to die activism}

\begin{abstract}
Based on ethnographic research among right to die activists, this article identifies and analyzes a puzzling conceptual divide that structures activists' arguments. On one hand, activists argue that a medically assisted death needs to be completely separate from the act of suicide. To ensure this, they employ the notion of "sound minds" in the context of "unsound bodies," focusing on the fundamental rationality of a voluntary decision-making process structured around choice. Their advocacy separates the act of medically assisted death from the irrationality of suicides, which ostensibly result from an "unsound mind." However, extensive ethnographic and interview data reveal multiple ambiguities and tensions in their attempt to distinguish between an officially sanctioned medically assisted death and an act of suicide, showing that activists implicitly recognize a porous border between these two acts. Analyzing these ambiguities explores a core tension between reformist and more radical sectors of right to die activism: while those aiming to change existing legislation argue for clearly defined eligibility criteria premised on terminal or incurable illness and accompanied by safeguards, more radical activists argue against this "medicalization of death" and advocate a more open position in which the right to die encompasses "rational suicide." This article will show that even after the legalization of medically assisted dying, these core tensions persist.
\end{abstract}

KEYWORDS - Death, right to die, medically assisted death, suicide.

\section{“El $99 \%$ de los suicidios son trágicos, nosotros luchamos por el $1 \%$ que queda." Unas "mentes sanas" en unos cuerpos enfermos y el activismo del derecho a la muerte}

RESUMEN - A partir de investigaciones etnográficas conducidas con activistas del derecho a la muerte, este artículo busca subrayar y analizar las divisiones de naturaleza conceptual que estructuran los argumentos de los activistas. Por un lado, algunos 
activistas sostienen que la muerte asistida por un médico debe ser diferenciada rigurosamente del acto suicida. Para ello, hacen referencia a la noción de la "mente sana" en un contexto en el que el cuerpo está enfermo, haciendo así un llamado al carácter fundamentalmente racional de un proceso decisional fundado sobre la voluntad en la decisión. Distinguen una tal decisión de los suicidios, no racionales, que son visiblemente el resultado de una "mente perturbada". Sin embargo, esbozando sobre una base de datos etnográficos considerable y en entrevistas, constatamos que los activistas reconocen también, implícitamente, una frontera permeable entre los dos actos. Varios puntos de tensión y de ambigüedad emergen, por cierto, cuando se trata de diferenciar la muerte asistida por un médico y oficialmente aprobada, y un acto de suicidio. En el análisis de la cuestión, el presente artículo busca explorar el núcleo de la tensión entre los reformistas y las ramas más radicales del activismo del derecho a la muerte. Si los que aspiran a modificar la constitución actual piden, entre otras salvaguardias, criterios de admisibilidad claramente definidos en función de enfermedades terminales e incurables, otros activistas más radicales se oponen a tales "medicalizaciones de la muerte", y defienden una posición más abierta en la que el derecho a la muerte engloba también a los "suicidas racionales". Finalmente, nuestro artículo mostrará que a pesar de la legalización de la muerte asistida por un médico, estos núcleos de tensiones persisten.

PALABRAS CLAVE - Muerte, derecho a la muerte, muerte asistida por un médico, suicidio. 